\title{
Effective Mass Density of Fluid-Solid Composites
}

\author{
Jun Mei, ${ }^{1}$ Zhengyou Liu, ${ }^{1, *}$ Weijia Wen, ${ }^{2}$ and Ping Sheng ${ }^{2}$ \\ ${ }^{1}$ Department of Physics, Wuhan University, Wuhan 430072, China \\ ${ }^{2}$ Department of Physics, The Hong Kong University of Science and Technology, Clear Water Bay, Kowloon, Hong Kong, China
}

(Received 15 August 2005; published 19 January 2006)

\begin{abstract}
We show through rigorous derivation and experimental support that the dynamic effective mass density of an inhomogeneous mixture, used in the prediction of wave velocities in the long wavelength limit, can differ from the static version - the volume average of the component mass densities. The physical reason for this difference is explained. The dynamic mass density expression, first derived by Berryman more than two decades ago, is shown to give a closer correspondence between the acoustic and electromagnetic metamaterials by allowing for negative mass densities at frequencies around resonances.
\end{abstract}

DOI: 10.1103/PhysRevLett.96.024301

PACS numbers: 43.35.+d, 43.20.+g, 43.40.+s

The effective mass density of a composite is one of the most basic quantities in the study of materials. It is common sense that the effective mass density of a mixture of materials should be their volume average, $D_{\text {eff }}=D_{V}=$ $D_{1}(1-f)+D_{2} f$, where $D_{1}$ and $D_{2}$ are the mass densities for the matrix and the inclusions, respectively, and $f$ is the filling ratio of the solid inclusions. This expression is denoted below as the volume-averaged mass density (VAMD). An important application of the composite effective mass density is in the prediction of wave velocities in the low frequency limit, where the relevant wavelength is much larger than the typical feature sizes in the composite. More than two decades ago, Berryman [1] derived a different effective mass density expression for the prediction of (fluid matrix-solid) composite wave properties in the long wavelength limit, based on the average $T$-matrix approach:

$$
\frac{D_{\text {eff }}-D_{1}}{(d-1) D_{\text {eff }}+D_{1}}=f \frac{D_{2}-D_{1}}{(d-1) D_{2}+D_{1}}
$$

where $d$ denotes the spatial dimensionality of the problem.

The Berryman effective mass density expression is noted to differ significantly from the intuitive VAMD, and for all the intervening years after the initial derivation it has remained a curiosity rather than extensively used, mainly owing to the lack of experimental support as well as to the strong sense that the intuitive VAMD must be correct, since otherwise it would be equivalent to stating the rather radical principle that the static mass density for a composite should be different from its dynamic mass density, even in the long wavelength limit. An additional objection to the Berryman expression is that the derivation treats the multiple scatterings inherent in the inhomogeneous system only in an averaged sense, and therefore not rigorously.

In this Letter, we show through rigorous derivation that the recent experimental evidence [2] has indeed provided strong support to the radical principle that the Berryman effective mass density expression should be the correct one to use in predicting the dynamic wave properties of fluid (matrix)-solid composites in the long wavelength limit, and that the VAMD is only a special case, obtainable from the Berryman expression when the components' mass density contrasts are small. The implications of this finding, especially relevant to the acoustic correspondence with the doubly negative response functions (electric and magnetic) of electromagnetic metamaterials, are presented.

The starting point of our considerations is the recent experiment [2] of Cervera et al., in which the sound velocity was measured in a two-dimensional phononic crystal [2-17] composed of regular arrays of rigid cylinders in air, confirming that in the low frequency (long wavelength) regime the sound propagates at subsonic velocity. In Fig. 1, we show the comparison of experimental results with the prediction of the usual effective medium theory (EMT) [18], in which the effective bulk modulus $B_{\text {eff }}$ is given by

$$
\frac{1}{B_{\text {eff }}}=\frac{1-f}{B_{1}}+\frac{f}{B_{2}},
$$

where $B_{1}=\lambda_{1}$ and $B_{2}=\lambda_{2}+\frac{2}{d} \mu_{2}$ are the bulk moduli for the liquid matrix and the solid inclusions, respectively, with $\lambda$ and $\mu$ being the Lamé constants. The effective mass density used in the effective medium theory prediction shown in Fig. 1 is given by VAMD. A very large discrepancy is seen. As the experiment is done with a regular array of solid cylinders, the system is amenable to accurate numerical predictions using the multiple scattering theory (MST) [17]. The MST-calculated results are shown as the solid triangles. Excellent agreement is seen.

Since the experiment was clearly in the long wavelength regime (with the wavelength at least 9 times the lattice constant), it becomes an intriguing question as to the form of the long wavelength (or the low frequency) limit of the exact theory, the prediction of which is shown to give good agreement with the experiment. This is because at the low frequency limit the dispersion relation for phononic crys- 


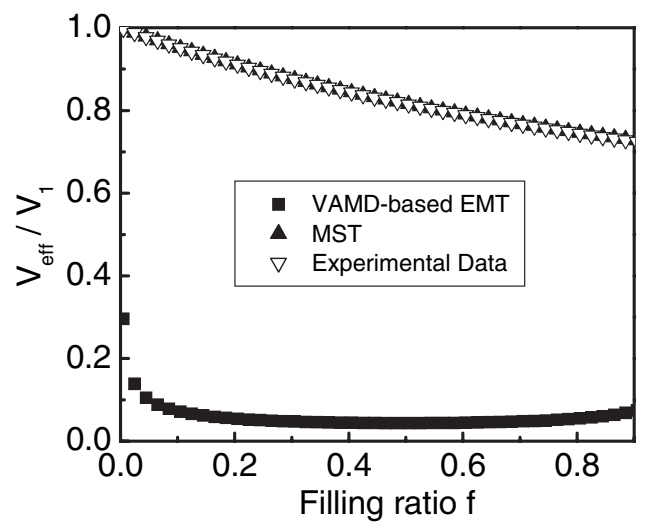

FIG. 1. The effective sound velocities calculated with VAMDbased EMT and with MST, respectively, compared with the experimentally measured effective sound velocity in a $2 \mathrm{D}$ phononic crystal composed of $\mathrm{Al}$ cylinders arranged in a hexagonal lattice in air, plotted as a function of the filling ratio of $\mathrm{Al}$ cylinders. $V_{1}$ is the sound velocity in air. Here the frequency of sound is $600 \mathrm{~Hz}$, the $\mathrm{Al}$ cylinders have a maximum diameter of $3.175 \mathrm{~cm}$, with a hexagonal lattice constant of $6.35 \mathrm{~cm}$. The wavelength of sound in air, $57 \mathrm{~cm}$, is thus much larger than either the cylinder diameter or the lattice constant. The wavelengths of sound in Al, $10.68 \mathrm{~m}$ for longitudinal wave and $5.19 \mathrm{~m}$ for transverse wave, are even larger. The use of VAMD in the effective medium prediction causes the large discrepancy seen in the figure.

tals has to be linear since one wavelength covers many periods of the structure, hence losing its wave resolution. Phononic crystals at low frequencies should therefore always possess well-defined effective material parameters. As the multiple scattering theory accounts for all the multiple scatterings in the system, it removes a principal objection to the Berryman effective mass density expression, which treats the multiple scattering only in an averaged sense.

MST [15-17] accounts fully for all the multiple scattering effects between any two scatterers, plus the vector character of elastic waves, without any approximation. For our particular case of 2D elastic MST in polar coordinates, the displacement of the incident wave on scatterer $i$ and the scattered wave (by the same scatterer) may be expressed, respectively, as

$$
\vec{u}_{i}^{\text {in }}\left(\vec{\rho}_{i}\right)=\sum_{n} a_{n}^{i} \vec{J}_{n}^{i}\left(\vec{\rho}_{i}\right), \quad \vec{u}_{i}^{\mathrm{sc}}\left(\vec{\rho}_{i}\right)=\sum_{n} b_{n}^{i} \vec{H}_{n}^{i}\left(\vec{\rho}_{i}\right),
$$

where the vector functions $\vec{J}_{n}(\vec{\rho})$ and $\vec{H}_{n}(\vec{\rho})$ are defined as

$$
\begin{aligned}
& \vec{J}_{n}(\vec{\rho})=\nabla\left[J_{n}\left(\alpha_{1} \rho\right) e^{i n \varphi}\right], \\
& \vec{H}_{n}(\vec{\rho})=\nabla\left[H_{n}\left(\alpha_{1} \rho\right) e^{i n \varphi}\right],
\end{aligned}
$$

with $\alpha_{1}$ being the wave number in the fluid matrix, $\vec{\rho}=$ $(\rho, \varphi)$ denoting the polar coordinates, and $J_{n}(x)$ and $H_{n}(x)$ on the right side of Eq. (4) denoting the $n$th order Bessel function and Hankel function of the first kind, respectively. Since the incident wave on scatterer $i$ comes from the scattered waves by all the scatterers except scatterer $i$, we have

$$
\vec{u}_{i}^{\text {in }}\left(\vec{\rho}_{i}\right)=\sum_{j \neq i} \sum_{n^{\prime \prime}} b_{n^{\prime \prime}}^{j} \vec{H}_{n^{\prime \prime}}^{j}\left(\vec{\rho}_{j}\right) .
$$

With the help of the addition theorem, we can prove that

$$
\vec{H}_{n^{\prime \prime}}^{j}\left(\vec{\rho}_{j}\right)=\sum_{n} G_{n^{\prime \prime} n}^{i j} \vec{J}_{n}^{i}\left(\vec{\rho}_{i}\right)
$$

where $G_{n n^{\prime}}^{i j}=G_{n n^{\prime}}\left(\vec{R}_{j}-\vec{R}_{i}\right)$ denotes the translation (from scatterer $i$ to scatterer $j$ ) coefficients, with $\vec{R}_{i(j)}$ denoting the position of scatterer $i(j)$. We refer to Ref. [17] for the precise definition of $G_{n n^{\prime}}(\vec{R})$. The expansion coefficients $A=\left\{a_{n}\right\}$ for the incident field and $B=\left\{b_{n}\right\}$ for the scattered field with reference to a given scatterer are related through the elastic Mie scattering matrix $T=\left\{T_{n n^{\prime}}\right\}$ for the scatterer by

$$
b_{n}=\sum_{n^{\prime}} T_{n n^{\prime}} a_{n^{\prime}}
$$

Substituting Eqs. (3), (6), and (7) into Eq. (5), we arrive at

$$
\sum_{j n^{\prime}}\left(\delta_{i j} \delta_{n n^{\prime}}-\sum_{n^{\prime \prime}} G_{n n^{\prime \prime}}^{i j} T_{n^{\prime \prime} n^{\prime}}^{j}\right) a_{n^{\prime}}^{j}=0 .
$$

For a periodic system, the normal modes of the system may be obtained by solving the following secular equation:

$$
\operatorname{det}\left|T_{n n^{\prime}}^{-1}-G_{n n^{\prime}}(\vec{k})\right|=0,
$$

where $G_{n n^{\prime \prime}}(\vec{k})=\sum_{R \neq 0} G_{n n^{\prime \prime}}(-\vec{R}) \exp (\overrightarrow{i k} \cdot \vec{R})$. By taking the low frequency limit of $\alpha_{1} \rightarrow 0$ and by retaining the dominant terms, Eq. (9) is simplified to a $3 \times 3$ matrix equation [19]:

$$
\operatorname{det}\left|\begin{array}{ccc}
\frac{D_{1}+D_{2}}{D_{1}-D_{2}}+\frac{x^{2} f}{1-x^{2}} & \frac{i x f}{1-x^{2}} & -\frac{f}{1-x^{2}} \\
-\frac{i x f}{1-x^{2}} & \frac{B_{2}}{B_{2}-B_{1}}+\frac{x^{2} f}{1-x^{2}} & \frac{i x f}{1-x^{2}} \\
-\frac{f}{1-x^{2}} & -\frac{i x f}{1-x^{2}} & \frac{D_{1}+D_{2}}{D_{1}-D_{2}}+\frac{x^{2} f}{1-x^{2}}
\end{array}\right|=0,
$$

in which $x=V_{\text {eff }} / V_{1}$ is the constant to be evaluated, while $f, B_{1}$, and $B_{2}$ are the filling ratio and the elastic moduli, respectively [20]. It can easily be verified that Eq. (10) is a quadratic equation of $x^{2}$, and by omitting the trivial root $x^{2}=1$, we obtain the root

$$
x^{2}=\frac{\left(D_{2}+D_{1}\right)-\left(D_{2}-D_{1}\right) f}{\left(D_{2}+D_{1}\right)+\left(D_{2}-D_{1}\right) f} \frac{B_{2}}{f B_{1}+(1-f) B_{2}} .
$$

By using the expression $V_{\text {eff }}=\sqrt{\frac{B_{\text {eff }}}{D_{\text {eff }}}}$, and the effective medium expression for $B_{\text {eff }}$, Eq. (2), we arrive at precisely the Berryman effective mass density in 2D. Equation (11) 
is noted to be valid for both the square and the hexagonal lattices. Hence, it is plausible that the Berryman effective mass density expression is generally valid for isotropic composites. This is the case especially since our derivation verifies, at the same time, the effective bulk modulus formula, Eq. (2), which is valid in general for isotropic composites consisting of solid inclusions in fluid [18].

As the static version of the effective mass density must be the VAMD (verifiable through simple weighing of the composite and its constituents, and measuring the volumes), the reason for the different (long wavelength) dynamic version can be found in the fact that, for wave properties, VAMD contains the implicit assumption of wave field homogeneity in the long wavelength limit. This assumption can be violated (even in the long wavelength limit) when there is a very large impedance mismatch between the two components, such as in the experiment of Cervera et al. In Fig. 2(a) we show the calculated wave field intensities, in color, for the relevant experiment. It is noted that the wave field is nearly zero inside the cylinders. Hence, it is almost impossible to have the condition for the validity of VAMD. The observed decrease in wave velocity can be ascribed to the wave paths' increased tortuosity. However, when the impedance mismatch is relatively moderate, e.g., when the mass density contrast is small, then the Berryman expression yields the VAMD. This is shown in Fig. 3, where the two expressions yield the almost identical effective mass density for the PMMA cylinders in water. For comparison with Fig. 2(a), we have also plotted the displacement field intensities for the PMMA-water system in Fig. 2(b), in which the wave field homogeneity is evident. As our derivation is obtained by taking the long wavelength limit of the rigorous scattering wave field solutions, it is not surprising that such a formula inherently accounts for the wave field inhomogeneities as they exist in reality. In Fig. 3 we have also examined the case of air cylinders in water, and it is seen that $D_{B}<D_{V}$ as well. It is easy to show from Eq. (1) that $D_{V}$ is an upper bound to $D_{B}$. We have verified that even in the case of fluid inclusions in a fluid matrix, the Berryman mass density expression is valid.

It should be noted that the effective bulk modulus expression, Eq. (2), derived via either the average $T$ matrix approximation [1] or the coherent potential approximation [18] (and verified through the low frequency limit of the MST), represents the low frequency limit of the $n=0$ angular scattering channel, whereas the $D_{B}$ expression is the low frequency limit of the $n=1$ channel. In the case examined above, the confinement of the wave amplitude in the interstitial space also implies that the effective bulk modulus is dominated by that of the fluid, just as predicted by Eq. (2), i.e., the two are consistent in this case. But even under general considerations $D_{B}$ and Eq. (2) should both be
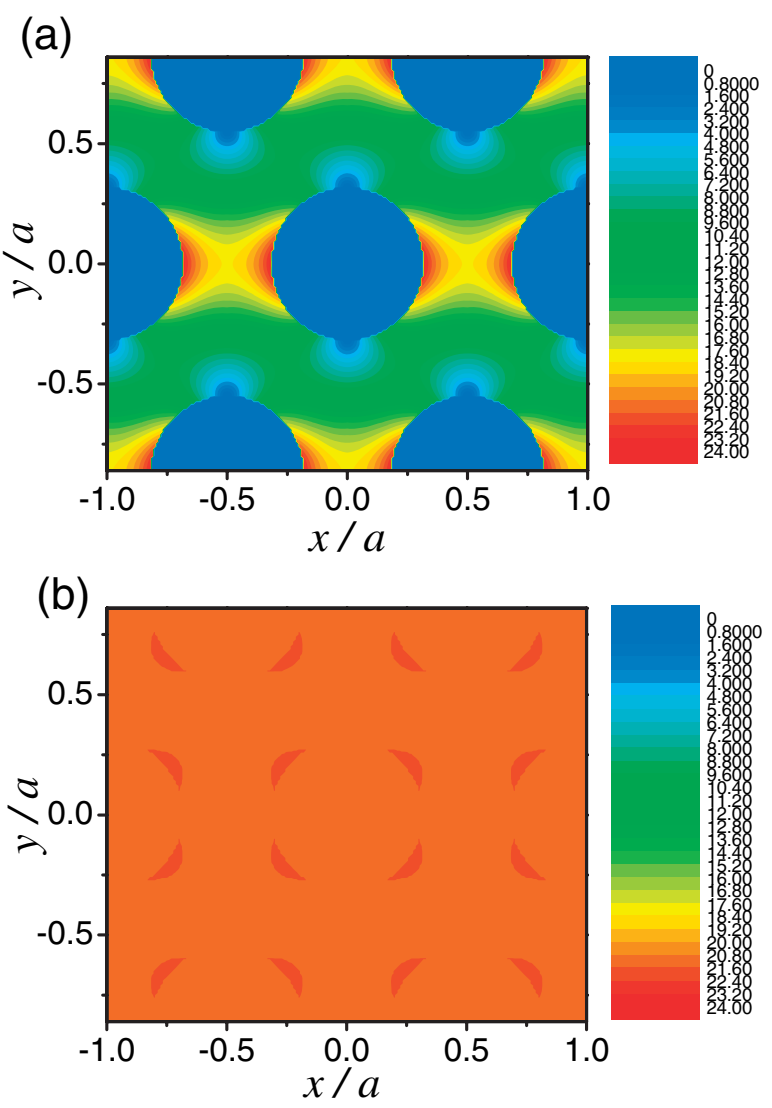

FIG. 2 (color). (a) MST-calculated displacement field intensities in the phononic crystal composed of $\mathrm{Al}$ cylinders arranged in a hexagonal lattice in air with the filling ratio of $\mathrm{Al}$ cylinders being 0.36. Blue is low field intensity, and red is high field intensity. The wave vector is along the $y$ direction, and $a$ is the lattice constant. It is seen that the wave amplitude is nearly zero inside the Al cylinders. Decreasing the frequency further does not alter this fact. (b) The same for PMMA cylinders in water. Wave field is seen to be much more homogeneous than that in (a).

valid, since they represent separate, yet at the same time parallel, wave scattering channels.

An important implication of our conclusion is in regard to the correspondence between acoustic $[21,22]$ and electromagnetic $[23,24]$ metamaterials. As VAMD can never be negative, it follows that if it is valid, then there can never be a one to one correspondence between the two classes of metamaterials in the effective medium limit. From Eq. (1), it is easily perceived that the expression allows a simple extension to the case when there is a $n=1$ resonance [19]:

$$
\frac{D_{\text {eff }}-D_{1}}{D_{\text {eff }}+D_{1}}=\frac{4 f}{i \pi\left(\alpha_{1} R\right)^{2}} S_{1},
$$

where

$$
S_{1}=T_{11}=-\frac{F_{1}\left[J_{1}\left(\alpha_{1} R\right)-\alpha_{1} R J_{2}\left(\alpha_{1} R\right)\right]+F_{2}\left[4 J_{2}\left(\alpha_{2} R\right)-\alpha_{1} R J_{3}\left(\alpha_{1} R\right)\right]}{F_{1}\left[H_{1}\left(\alpha_{1} R\right)-\alpha_{1} R H_{2}\left(\alpha_{1} R\right)\right]+F_{2}\left[4 H_{2}\left(\alpha_{2} R\right)-\alpha_{1} R H_{3}\left(\alpha_{1} R\right)\right]}
$$




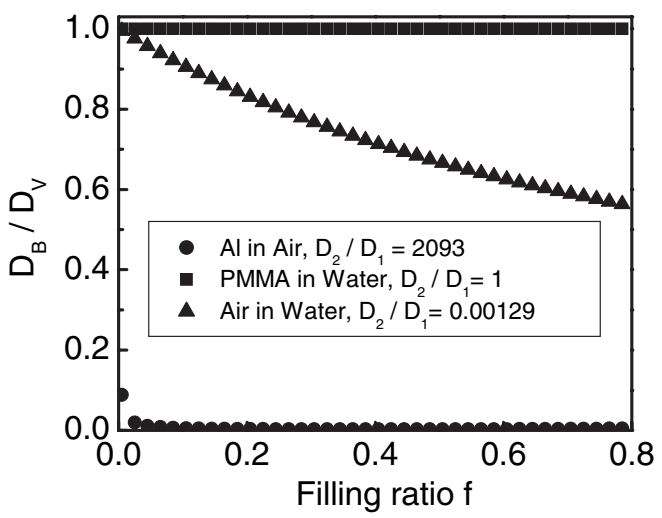

FIG. 3. The Berryman effective mass density normalized with the VAMD for three different phononic crystals composed of $\mathrm{Al}$ cylinders in air, PMMA cylinders in water, and air cylinders in water, plotted as a function of the filling ratio $f$ of the cylinder inclusions. $D_{1}$ is the mass density of the matrix, and $D_{2}$ is the mass density of inclusions.

is the Mie scattering coefficient for the angular momentum $n=1$ channel for a single cylinder with radius $R$, while $F_{1}$ and $F_{2}$ are functions of variables $R, \alpha_{1}, \alpha_{2}$, and $\beta_{2}$, with $\alpha_{1}, \alpha_{2}$, and $\beta_{2}$ being the longitudinal wave number in the background fluid, and longitudinal and transverse wave numbers in the solid scatterers, respectively. From Eq. (12) it is easily seen that the effective mass density can be negative for $\left|S_{1}\right|>\frac{\pi\left(\alpha_{1} R\right)^{2}}{4 f}$. But if there is a resonance in the $n=1$ channel, the modulus of the scattering amplitude can be large, so the effective mass density can turn negative as well. This fact was noted in Ref. [22], but the justification for using the Berryman mass density expression was lacking in that case.

In conclusion, we find the Berryman effective mass density to be the correct one for predicting dynamic wave properties, regardless of the impedance mismatch between the components. Our conclusion is based on taking the long wavelength limit of the rigorous multiple scattering theory, and on excellent agreement with the experimental result of Cervera et al.

This work was supported by the National Science Foundation of China (Grants No. 50425206 and No. 10174054), the Hong Kong Research Grant Council (Grant No N_HKUST605/04), and Doctoral Research Foundation of Ministry of Education of China (Grant No. 20020486013).

*Electronic address: zyliu@whu.edu.cn

[1] James G. Berryman, J. Acoust. Soc. Am. 68, 1809 (1980); 68, 1820 (1980). Please note that the $(d-1)$ factor in Eq. (1b) is situated differently from the well-known Maxwell-Garnett formula. This is because the dielectric constant $\varepsilon$ corresponds with $1 / \rho$.

[2] F. Cervera, L. Sanchis, J. V. Sánchez-Pérez, R. MartínezSala, C. Rubio, F. Meseguer, C. López, D. Caballero, and J. Sánchez-Dehesa, Phys. Rev. Lett. 88, 023902 (2002);
Lorenzo Sanchis, Andreas Håkansson, Francisco Cervera, and José Sánchez-Dehesa, Phys. Rev. B 67, 035422 (2003).

[3] M. M. Sigalas and E. N. Economou, Solid State Commun. 86, 141 (1993); Europhys. Lett. 36, 241 (1996).

[4] M.S. Kushwaha, P. Halevi, L. Dobrzynski, and B. Djafari-Rouhani, Phys. Rev. Lett. 71, 2022 (1993); M. S. Kushwaha, P. Halevi, G. Martínez, L. Dobrzynski, and B. Djafari-Rouhani, Phys. Rev. B 49, 2313 (1994).

[5] R. Mártinez-Sala, J. Sancho, J. V. Sánchez-Pérez, J. Llinares, and F. Meseguer, Nature (London) 378, 241 (1995); J. V. Sánchez-Pérez, D. Caballero, R. MártinezSala, C. Rubio, J. Sánchez-Dehesa, F. Meseguer, and F. Gálvez, Phys. Rev. Lett. 80, 5325 (1998).

[6] C. Goffaux, J. Sánchez-Dehesa, A. L. Yeyati, Ph. Lambin, A. Khelif, J. O. Vasseur, and B. Djafari-Rouhani, Phys. Rev. Lett. 88, 225502 (2002); A. Khelif, A. Choujaa, S. Benchabane, B. Djafari-Rouhani, and V. Laude, Appl. Phys. Lett. 84, 4400 (2004).

[7] F. R. Montero de Espinosa, E. Jiménez, and M. Torres, Phys. Rev. Lett. 80, 1208 (1998); M. Torres, F. R. Montero de Espinosa, D. García-Pablos, and N. García, Phys. Rev. Lett. 82, 3054 (1999); M. Torres, F. R. Montero de Espinosa, and J. L. Aragón, Phys. Rev. Lett. 86, 4282 (2001).

[8] C. M. Soukoulis, S. Datta, and E. N. Economou, Phys. Rev. B 49, 3800 (1994).

[9] J. O. Vasseur, P. A. Deymier, B. Chenni, B. DjafariRouhani, L. Dobrzynski, and D. Prevost, Phys. Rev. Lett. 86, 3012 (2001).

[10] Y. Tanaka and S. Tamura, Phys. Rev. B 58, 7958 (1998).

[11] Tsung-Tsong Wu, Zi-Gui Huang, and S. Lin, Phys. Rev. B 69, 094301 (2004).

[12] You-Yu Chen and Zhen Ye, Phys. Rev. Lett. 87, 184301 (2001).

[13] A. A. Krokhin, J. Arriaga, and L. N. Gumen, Phys. Rev. Lett. 91, 264302 (2003).

[14] M. Kafesaki, R. S. Penciu, and E. N. Economou, Phys. Rev. Lett. 84, 6050 (2000).

[15] I. E. Psarobas, N. Stefanou, and A. Modinos, Phys. Rev. B 62, 278 (2000); I.E. Psarobas, N. Stefanou, and A. Modinos, Phys. Rev. B 62, 5536 (2000).

[16] Z. Liu, C. T. Chan, P. Sheng, A. L. Goertzen, and J.H. Page, Phys. Rev. B 62, 2446 (2000).

[17] Jun Mei, Z. Liu, J. Shi, and D. Tian, Phys. Rev. B 67, 245107 (2003).

[18] Ping Sheng, Introduction to Wave Scattering, Localization, and Mesoscopic Phenomena (Academic Press, New York, 1995), Chap. 3.

[19] The derivation is straightforward but tedious. It will be published elsewhere.

[20] The shear wave excitations are fully accounted for in the MST, but at low frequency expansions they appear only in orders higher than $n=0,1$ and are negligible.

[21] Zhengyou Liu, C. T. Chan, and Ping Sheng, Phys. Rev. B 71, 014103 (2005).

[22] Jensen Li and C. T. Chan, Phys. Rev. E 70, 055602 (2004).

[23] V. G. Veselago, Sov. Phys. Usp. 10, 509 (1968).

[24] J. B. Pendry et al., Phys. Rev. Lett. 76, 4773 (1996); J. B. Pendry et al., IEEE Trans. Microw. Theory Tech. 47, 2075 (1999). 